\title{
Vaccination and Longevity
}

Between 1940 and 1998 life expectancy at birth in Brazil increased by around 30 years, mainly as the result of the reduction of deaths due to vaccine-preventable infectious diseases. The vaccination of children, which reduced not only cases of illness but also the circulation of infectious agents among the population, had a positive impact on the health of adults and the elderly (collective protection).

The initial decline in mortality, substantially impacted by the reduction in deaths from infectious diseases and the aging of the population, has since seen an increase in cases of chronic and degenerative diseases. Diseases have changed from an acute process that ends in cure or death to a chronic state where people suffer for long periods of life and which when not adequately controlled can generate disabilities, significantly compromising the quality of life of these individuals.

In the process of Latin American epidemiological transition, unlike in developed nations, the increase in chronic and degenerative diseases (typical of aging) has overlapped with infectious diseases which are not yet fully controlled in these countries. In this way, the increase in the number of deaths due to diseases of the circulatory system, among others, has resulted in a scenario in which the proportion of infectious and parasitic diseases differs considerably from that of developed countries. This "double burden of disease" squeezes the already scarce resources of public health to the limit.

Moreover, while celebrating the extra years gained, it must be recognized that greater longevity without quality of life is a hollow prize, or in other words, health expectancy is as or more important than life expectancy. The prevention of infectious diseases is part of this situation, allowing the reduction of morbidity.

Elderly persons are among the most vulnerable to the serious outcomes caused by infectious diseases: hospitalizations; deaths; decompensation due to underlying illnesses such as diabetes, heart disease and chronic lung disease; increased risk for myocardial infarction or stroke; sequelae that negatively impact quality of life on the loss of independence and the incapacitation of the individual. Vaccines can be considered important tools for the successful adaptation of the body to occurrences that threaten its functions and viability in aging.

Current gains in our understanding of immunizations have allowed a series of innovations, with the availability of new vaccines and strategies geared toward the health of the elderly. This knowledge, combined with the changing epidemiology of infectious agents, has led to a rapid cycle of updates in vaccination schedules, to align current clinical practice with scientific progress. 
The Brazilian Immunization (SBIm) and Geriatrics and Gerontology (SBGG) societies recommend the routine vaccination of the elderly with the following vaccines: influenza, Pneumococcal 13-valent conjugate, 23-valent pneumococcal polysaccharide, herpes zoster, hepatitis B and pertussis (whooping cough).

While vaccination is one of the most effective public health measures for preventing disease, vaccine coverage rates in the adult population remain below target, even when vaccines are offered for free. The main barriers to adult vaccination are thought to be misguided beliefs and low awareness among patients and insufficient knowledge and negative attitudes on the part of doctors. The lack of medical prescription is described in literature as the main reason for non-vaccination among adults.

Brazil has aged and policies to increase adult immunization are required. These should seek to: include the theme of immunization in medical education programs and the training of all health professionals; engage civil society in raising public awareness; establish or improve surveillance systems to determine and monitor the burden of vaccine-preventable diseases in adults; bring together different recommendations and protocols; create immunization records for adults and integrate these into electronic medical records.

Isabella Ballalai

President of the Brazilian Immunization Society (SBIm) 